06,11

\title{
О механизме повышения температуры фазового перехода в сегнетоактивных нанокомпозитах
}

\author{
(C) В.Н. Нечаев ${ }^{1}$, А.В. Висковатых ${ }^{2, \uparrow}$ \\ ${ }^{1}$ Воронежский государственный технический университет, \\ Воронеж, Россия \\ ${ }^{2}$ Белгородский государственный национальный исследовательский университет, \\ Белгород, Россия \\ ฯ E-mail: ostrogvisk@mail.ru
}

(Поступила в Редакцию 14 декабря 2015 г.

В окончательной редакции 20 мая 2016 г.)

Путем численного анализа нелинейной системы уравнений показано, что в нанокомпозите сегнетоэлектрик-диэлектрик возможно повышение температуры сегнетоэлектрического фазового перехода при наличии переходного слоя на границе сегнетоэлектрик-диэлектрик. Исследованы распределение параметра порядка в сегнетоэлектрической частице и влияние толщины переходного слоя на температуру Кюри композита.

Значительно возросшая в последние годы интенсивность исследований нанокомпозиционных материалов обусловлена, с одной стороны, широким применением их в различных областях техники, в электронике, оптике, медицине и т. д. вследствие возможности целенаправленного формирования интегральных свойств композитов в процессе их получения путем варьирования размеров, формы и других параметров входящих в их состав компонентов $[1,2]$. С другой стороны, важен фундаментальный аспект, связанный с физикой наноразмерных частиц. Для сегнетоактивных композитов основными изучаемыми свойствами являются температура фазового перехода в сегнетоактивной компоненте и их диэлектрическая проницаемость. Имеющиеся экспериментальные данные по этому вопросу зачастую противоречивы, отсутствует также единое мнение относительно формирования свойств сегнетоактивных наносистем и ведущих механизмов, их определяющих. Так, рядом авторов $[3,4]$ наблюдалось уменьшение температуры фазового перехода в композиционных материалах с наноразмерными включениями по сравнению с температурой массивного сегнетоэлектрика. В то же время эксперимент [5] показал заметное повышение температуры Кюри композитов с внедренными частицами $\mathrm{KH}_{2} \mathrm{PO}_{4}$ и, наоборот, ее понижение для композитов с частицами $\mathrm{CsH}_{2} \mathrm{PO}_{4}$ при уменьшении их диаметра. Авторы [6] сообщают о повышении температуры фазового перехода системы триглицинсульфат (ТГС) $-\mathrm{Al}_{2} \mathrm{O}_{3}$ по сравнению с наблюдаемой для массивного кристалла ТГС. Такое несколько неожиданное поведение исследуемой системы, авторы [6] связывают с появлением новых взаимодействий химической природы на границе сегнетоэлектрика и диэлектрической матрицы либо с трансформацией существующих связей в сегнетоэлектрическом кристалле вблизи границы раздела компонентов. Оценки толщины трансформированного слоя в работе не проводилось, но логично предположить, что она должна составлять величину порядка нескольких межатомных расстояний.
В работе [7] рассмотрен частный случай бесконечно малой толщины переходного трансформированного слоя, а также его учет через поверхностную составляющую термодинамического потенциала рассматриваемой композиционной системы сегнетоэлектрик-диэлектрик.

Целью настоящей работы является исследование влияния трансформированного слоя сегнетоэлектрика конечной толщины на границе с диэлектрической матрицей на повышение температуры фазового перехода нанокомпозиционного сегнетоактивного материала.

В качестве объекта исследования выбрана нанокомпозиционная система, структурно-функциональной единицей которой является куб с ребром $L$ из диэлектрического материала $\left(\mathrm{SiO}_{2}\right)$ и расположенным в его центре сегнетоэлектрическим выделением (ТГС) в форме эллипсоида вращения с полуосями $a$ и $b(b>a)$. Предположим, что между сегнетоэлектриком и матрицей имеется переходный слой толщиной $l_{s}$, который вследствие изменения химических связей вблизи границы раздела будет иметь параметры, отличные от параметров сегнетоэлектрической частицы. К числу таких параметров относятся, в частности, температура Кюри, коэффициенты $\alpha, \beta$ в разложении свободной энергии в духе Ландау.

Пусть сегнетоэлектрическое включение в диэлектрической матрице представляет собой одноосный сегнетоэлектрик с сегнетоактивной осью, совпадающей с полуосью $b$ и параллельной координатной оси $O z$. Ребра структурно-функциональной ячейки совпадают с направлением ортов прямоугольной координатной системы. Для сегнетоэлектрической частицы распределение вектора поляризации $\mathbf{P}=\left\{0,0, P_{z}\right\}$ и напряженности электрического поля $\mathbf{E}=-\nabla \varphi$ полностью описывается системой уравнений, полученной в результате варьирования термодинамического потенциала системы сегнетоэлектрическая частица-диэлектрическая матрица с учетом диэлектрических полей и поверхностной составляющей термодинамического потенциала [8]. Система 
уравнений для $P_{z}$ и $\varphi$ имеет вид

$$
\left\{\begin{array}{l}
-\kappa \Delta P_{z}-\alpha P_{z}+\beta P_{z}^{3}=-\frac{\partial \varphi}{\partial z}, \\
\Delta\left(\varepsilon_{1} \varphi\right)=4 \pi \frac{\partial P_{z}}{\partial z}
\end{array}\right.
$$

Здесь $P_{z}-z$-я компонента вектора поляризации $P$, играющая роль параметра порядка при фазовом переходе, $\varphi$ - электрический потенциал, $\varepsilon_{1}$ - вклад в диэлектрическую проницаемость электронной подсистемы включения, $\alpha, \beta-$ коэффициенты Ландау, $\alpha=\alpha_{0}\left(T_{0}-T\right)$, $T_{0}$ - температура фазового перехода бесконечного сегнетоэлектрика, $\kappa=c^{2}-$ корреляционная постоянная, $c$ - межатомное расстояние.

Для переходного слоя толщиной $l_{s}$ справедлива система уравнений (1), (2) с коэффициентами Ландау $\alpha^{(s)}, \beta$; $\alpha^{(s)}=\alpha_{0}\left(T_{0}^{(s)}-T\right), T^{(s)}-$ температура фазового перехода сегнетоэлектрика в переходном слое.

Для диэлектрической матрицы справедливо уравнение Лапласа

$$
\Delta\left(\varepsilon_{2} \varphi\right)=0,
$$

где $\varepsilon_{2}$ - диэлектрическая проницаемость матрицы.

Граничные условия к уравнениям (1)-(3) на поверхности Г между переходным слоем и матрицей и на поверхности $\Gamma^{(s)}$ между сегнетоэлектриком и переходным слоем имеют вид

$$
\begin{gathered}
\left.\left(\frac{\partial P_{z}}{\partial \mathbf{n}}-\frac{1}{s} P_{z}\right)\right|_{\Gamma, \Gamma^{(s)}}=0 \\
\left.\varphi^{E F}\right|_{\Gamma, \Gamma^{(s)}}=\left.\varphi^{D E}\right|_{\Gamma, \Gamma^{(s)}}, \\
\left.\varepsilon_{2} \frac{\partial \varphi^{D E}}{\partial \mathbf{n}}\right|_{\Gamma, \Gamma^{(s)}}=\left.\left(\varepsilon_{1} \frac{\partial \varphi^{F E}}{\partial \mathbf{n}}-4 \pi \mathbf{P n}\right)\right|_{\Gamma, \Gamma^{(s)}},
\end{gathered}
$$

где $s$ - параметр, характеризующий взаимодействие сегнетоэлектрической частицы с матрицей, $\mathbf{n}-$ единичный вектор внешней нормали к поверхности Г.

На внешних границах структурной ячейки композита рассматривались нулевые условия Дирихле для параметра порядка и для электрического потенциала.

Нелинейная краевая задача (1)-(6) решена численно методом конечных элементов в пакете прикладных программ Comsol Multiphysics со следующими параметрами для сегнетоэлектрического включения (ТГС) и матрицы: $T_{0}=322 \mathrm{~K}, T_{0}^{(s)}=330 \mathrm{~K}, \varepsilon_{1}=5, \varepsilon_{2}=15, a=9 \mathrm{~nm}$, $b=45 \mathrm{~nm}, L=150 \mathrm{~nm}$. Параметр $s$ в (4) рассматривался в виде $s \rightarrow \infty$, что соответствует граничному условию Неймана для параметра порядка при фазовом переходе и отражает минимальное влияние матрицы на формирование полярной фазы в сегнетоэлектрической наночастице. Получены профили распределения поляризации в структурно-функциональной единице композиционного материала (рис. 1). На рис. 2 показаны зависимости температуры фазового перехода рассматриваемой нанокомпозитной структуры от толщины переходного слоя,

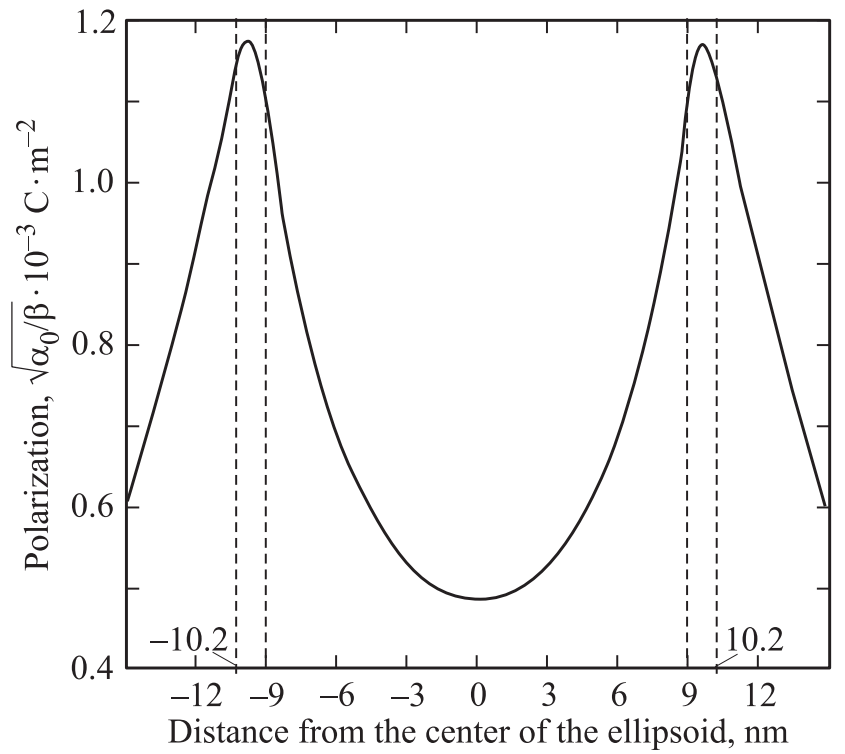

Рис. 1. Профиль распределения поляризации вдоль малой оси сегнетоэлектрического эллипсоида при $T=324 \mathrm{~K}, a=9 \mathrm{~nm}$, $b=45 \mathrm{~nm}, d=1.2 \mathrm{~nm}$.

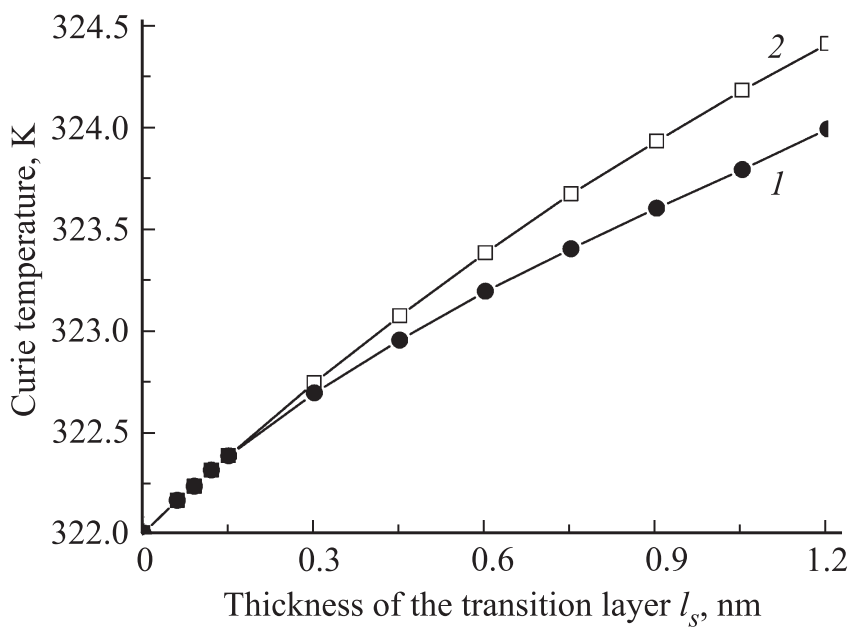

Рис. 2. Зависимость температуры фазового перехода сегнетоэлектрической частицы $(a=9 \mathrm{~nm}, b=45 \mathrm{~nm})$ от толщины переходного слоя $l_{s}$ в рамках моделей с переходным слоем конечной толщины $(1)$ и со слоем бесконечно малой толщины (2).

полученные в рамках модели с переходным слоем конечной толщины, в сравнении с результатами для бесконечно тонкого слоя. Как видно из полученных результатов, температура фазового перехода системы оказывается выше при наличии переходного трансформированного слоя по сравнению с соответствующей температурой объемного сегнетоэлектрика. Естественно, что величина смещения температуры фазового перехода зависит от толщины $l_{s}$ трансформированного слоя и температуры Кюри этого слоя $T_{0}^{(s)}$. Как видно из результатов (рис. 1,2), при повышении температуры Кюри в пере- 
ходном слое (на $8 \mathrm{~K}$ ) при толщине слоя $l_{s}=1.2 \mathrm{~nm}$, что соответствует четырем межатомным расстояниям, температура фазового перехода композита увеличилась на $2 \mathrm{~K}$ относительно температуры Кюри бесконечного сегнетоэлектрика. При увеличении толщины переходного слоя температурный интервал существования полярной фазы увеличивается, очевидно асимптотически приближаясь к температуре фазового перехода трансформированного слоя.

Следует отметить, что при уменьшении параметра $s$ в (4) наблюдаемое повышение температуры Кюри будет проявляться слабее из-за влияния на параметр порядка со стороны матрицы. В общем случае влияние граничных условий на температуру Кюри сегнетоактивных наносистем рассмотрено в предыдущих работах $[1,2,8]$.

Зависимость температуры Кюри в рамках бесконечно тонкого переходного слоя получена в рамках модели [7], согласно которой граничные условия для поляризации имеют вид

$$
\left.\left(\kappa \frac{\partial P_{z}}{\partial \mathbf{n}}-\alpha_{s} P_{z}+\beta_{s} P_{z}^{3}\right)\right|_{\Gamma}=0,
$$

где $\alpha_{s}=\alpha_{0}\left(T_{0}^{(s)}-T\right) l_{s}, \beta_{s}=\beta l_{s}$ - коэффициенты разложения термодинамического потенциала переходного слоя бесконечно малой толщины $l_{s}$ в духе Ландау, $T_{0}^{(s)}$ — температура Кюри поверхностного слоя.

Толщина поверхностного слоя $l_{s}$ выражается через известные параметры [9]:

$$
l_{s}=\frac{1}{\varsigma} \frac{\xi^{2}}{\delta}
$$

где $\xi=\sqrt{2 \kappa / \alpha}-$ корреляционная длина, $\delta-\kappa / \alpha_{s}-$ длина экстраполяции, $s$ - коэффициент, учитывающий форму частицы.

При толщине переходного слоя $l_{s} \leq 0.15 \mathrm{~nm}$ результаты, полученные в рамках моделей с переходным слоем конечной толщины и с переходным слоем сегнетоэлектрика бесконечно малой толщины, хорошо согласуются. При увеличении толщины переходного трансформированного слоя сегнетоэлектрика температура фазового перехода, определенная в рамках модели с переходным слоем бесконечно малой толщины, оказывается несколько выше соответствующей температуры, полученной в рамках модели со слоем конечной толщины. По мере роста $l_{s}$ это различие увеличивается.

Среди других обсуждаемых в литературе факторов, способствующих расширению области существования полярной фазы в композите, следует отметить влияние электрического взаимодействия сегнетоэлектрических выделений $[10,11]$ на температуру фазового перехода. При этом в [10] сегнетоэлектрические выделения рассматривались как полярные наночастицы, а в [11] они моделировались электрическими точечными диполями. В работе [12] было показано, что влияние всестороннего сжатия со стороны матрицы, возникающее в результате различных коэффициентов температурного расширения компонентов композита, при определенных условиях также способствует повышению температуры Кюри. При анализе экспериментальных данных, естественно, все перечисленные механизмы, включая рассмотренный в настоящей работе, должны приниматься во внимание.

\section{Список литературы}

[1] В.Н. Нечаев, А.В. Шуба, А.В. Висковатых. Изв. РАН. Сер. физ. 74, 1273 (2010).

[2] В.Н. Нечаев, А.В. Висковатых. Вестн. ВГтУ 7, 12.1, 54 (2011).

[3] О.А. Караева, Л.Н. Коротков, А.А. Набережнов, Е. Rysiakiewicz-Pasek. ФTT 51, 1304 (2009).

[4] С.В. Барышников, Е.В. Чарная, А.Ю. Милинский, A.Ю. Гойхман, C. Tien, M.K. Lee, L.J. Chang. ФTT 55, 987 (2013).

[5] Л.Н. Коротков, В.А. Тарнавич, Т.Н. Короткова, Р.Р. Левицкий, С.И. Сороков, А.С. Вдович. В сб.: Тез. докл. XIX Bсерос. конф. по физике сегнетоэлектриков. М. (2011). C. 111

[6] Н.Г. Поправко, Ю.С. Тучина. Вестн. ТГТУ 18, 731 (2012).

[7] В.Н. Нечаев, А.В. Шуба. ФТТ 56, 949 (2014).

[8] В.Н. Нечаев, А.В. Шуба, А.В. Висковатых. В сб.: Материалы VI Междунар. семинара „Физико-математическое моделирование систем“. ВГТУ, Воронеж (2009). Т. 1. С. 38.

[9] В.Н. Нечаев, А.В. Шуба, А.В. Висковатых. Изв. вузов. Физика 58, 114 (2015).

[10] В.Н. Нечаев, А.В. Висковатых. В сб.: Материалы VII Междунар. семинара „Физико-математическое моделирование систем“. ВГТУ, Воронеж (2011). Т. 3. С. 15.

[11] С.В. Барышников, Е.В. Чарная, Ю.А. Шацкая, А.Ю. Милинский, М.И. Самойлович, D. Michel, C. Tien. ФТТ 53, 1146 (2011).

[12] В.Н. Нечаев, А.В. Висковатых. ФТТ 56, 1930 (2014). 